\title{
Personal care workers in Australian aged care: Retention and turnover intentions
}

\section{Abstract}

Aim(s) - This study examined factors influencing personal care workers' intentions to stay or leave Australian aged care employment, especially for older workers.

Background - Retention of personal care workers is particularly important in aged care, as they provide the majority of the direct care via community aged care or long-term aged care environments. However, limited research has examined what drives their turnover and retention.

Method(s) - A survey was conducted during 2012, collecting 206 responses from workers within community and long-term aged care in four organizations in Australia.

Results - Perceived supervisor support, on-the-job embeddedness and area of employment were identified as predictors of both intentions to stay and leave, although the relationship strength differed. Community care workers were more likely to stay and reported more supervisor support than longterm care workers. Unexpectedly, age and health status were not predictors of staying or leaving.

Conclusion(s) - While there are similarities between retention and turnover motivators, there are also differences. Within a global context of health worker shortages, such new knowledge is keenly sought to enhance organizational effectiveness and sustain the provision of quality aged care.

Implications for Nursing Management - Retention strategies for older workers should involve increasing supervisor support, and seeking to embed workers more fully within their organization.

Keywords: retention, turnover, personal care workers, workforce, aged care 


\section{Introduction}

Population ageing brings with it many challenges associated with providing quality health and aged care services in most OECD countries, including Australia (OECD 2005; Productivity Commission 2005). Such challenges include the changing patterns of disease in older people and increasing levels of disability, chronicity and frailty (Australian Institute of Health and Welfare (AlHW) 2010). Simultaneously, service providers face increasing demands for quality health and aged care services. In Australia, qualified and experienced workers are needed to provide care and support for residents or clients in both long-term aged care (LTAC) and community aged care (CAC) settings (Department of Health and Ageing (DoHA) 2005). While much research has been conducted on the retention and turnover of nurses, limited research has examined personal care workers. In Australia, personal care workers are those who assist clients with their daily personal care routine such as showering, toileting, and providing assistance with meals and make up $68 \%$ of the workers within LTAC environments and $81 \%$ of workers within CAC environments (King et al. 2012). Therefore, there is an urgent need to devise evidence-based strategies to assist healthcare organizations to retain their workforce.

Retaining older workers in Australia is particularly important as the average retirement age in 2011 was 53 years (Australian Bureau of Statistics 2011). Within an aged care context, however, this need is even more apparent as the median age of personal care workers was 47 years (LTAC setting), and 50 years (CAC setting) (King et al. 2012). Thus, research informing retention strategies for older personal care workers, aged over 45 years, in aged care is needed.

Research examining employee retention and turnover found that older workers report higher intentions to stay (Reitz et al. 2010) and lower intentions to leave, than younger workers (Cheung 2004) across multiple international settings. While these findings suggest similarities between the causes of retention and turnover outcomes for older workers, most studies examined the drivers for either retention or turnover (Eley et al. 2010). The few studies (e.g., Chan and Morrison 2008) that examined both outcomes reported mixed results. For example, one quantitative study examining Singapore hospital nurses found similar drivers for intentions to stay and leave (Chan and Morrison 2008). In contrast, an American qualitative study by Mittal et al. (2009) found different drivers of aged care workers' intentions to stay and leave. Therefore, our research aimed to clarify the retention and 
turnover influences upon employees, with a focus on older workers, as research has found that older workers often experience age discrimination in the workforce (Encel and Studencki 2004).

Previous research examining turnover and retention has identified several variables that are correlated with both outcomes. These include demographic variables (age, health, financial circumstances; Department of the Treasury 2004; Phillipson 2004) and employment-related variables (area of employment, job embeddedness and supervisor support), yet little attempt has been made to clarify whether the same factors affect the two outcomes in the same way. Additionally, no research was found examining differences in retention and turnover drivers for LTAC and CAC workers. Therefore, this research extends the turnover and retention literature by examining, in a single study, the influences of a range of factors upon older personal care workers' intentions to stay and leave. To do this, personal care workers from both LTAC and CAC settings were investigated. Our study therefore examined three research questions.

1. What factors influence personal care workers' intentions to stay and/or leave?

2. What are the similarities and differences between the factors predicting older personal care workers' intentions to stay and/or their intentions to leave?

3. Do the factors predicting work participation intentions differ between LTAC and CAC employees?

\section{Background}

The Australian aged care sector provides short-term, medium-term and long-term care to people aged over 65 (DoHA 2004) living in either LTAC or CAC settings. LTAC is provided for those unable to live independently in their own home, while temporary LTAC (respite) is provided to carers (of older people), who may themselves have health conditions and to alleviate carer strain (AlHW 2010). In contrast, CAC services aid older people to stay in their own homes and maintain independent lifestyles within their community, while still accessing necessary care (DoHA 2011). In total, the aged care sector provides services to over 209,000 people in Australia (AlHW 2012). In order to provide these services, sufficient experienced and qualified employees are required. 
The Australian aged care workforce consists of qualified nursing staff - Registered Nurses, Enrolled Nurses, Enrolled Endorsed Nurses - and unregulated care workers - Assistants in Nursing and Personal Care Workers (King et al. 2012). While the scope of professional practice for each of these job titles is clearly defined by regulatory and industry bodies and is similar across LTAC and CAC settings, the culture, team environment and support differ. That is, in LTAC environments, a team of carers manages a client's care at any one time within close proximity to each other, while in CAC environments, the team is a "virtual" one, with team members providing individual care to clients in their homes (Productivity Commission 2011). Given the different physical and organizational environments in which they work, it is reasonable to expect that CAC and LTAC carers will have different drivers of retention and turnover. More specifically, as elaborated below, the intentions of those working within LTAC settings may be more strongly affected by on-the-job embeddedness factors, while those working in CAC settings may be more highly affected by off-the-job embeddedness factors.

\section{Employee turnover and retention}

Research has consistently found that employees' intentions to leave are the strongest predictors of actual turnover behavior (McCarthy et al. 2007; Morrell et al. 2006). This is because employees who have left are often difficult to locate, and thus their reasons for leaving cannot be ascertained, (Lambert and Hogan 2009), instead researchers frequently use turnover intentions as a proxy for actual turnover behaviour. In addition to the extensive research examining employees' intentions to leave, there is a recent and growing body of literature investigating employees' intentions to stay, with an emphasis on either younger and older workers, as an employee's age has been identified as an indicator of their intention to remain employed (Tourangeau and Cranley 2006). Prior to the Global Financial Crisis (GFC) in 2008, employers were generally pessimistic about employing older workers and early retirement was common (Mountford 2011). However, since the GFC, such pessimism has diminished to meet labor shortages. Simultaneously, in Australia following the GFC, according to O'Loughlin et al. (2010), 36.5\% of workers aged 50-64 had decided to postpone their retirement plans. Ferres et al. (2003) found that younger workers had higher intentions to leave than older workers, supporting Palese et al. (2006) who found that older nurses are more permanent, have lower levels of turnover and higher levels of affective commitment to their organization. 
Many organizational factors have been proposed to explain older employees' retention and turnover. For example, Shacklock and Brunetto (2011a) found that the importance of work itself and management effects (access to flexible working arrangements, supervisor support) were indicators of older employees' intentions to continue working. For older nurses, attachment to the work itself was identified as a key driver of remaining working, along with the relationships built with others at work (Shacklock and Brunetto 2011b). Further, a survey of older employees found the attractiveness of work hours, and the financial need to stay, the job itself, and job security/stability were significant to their intentions to continue working (Radford et al. 2012).

Personal factors that potentially explain intentions to stay and leave include employee age, health, and financial circumstances (Productivity Commission 2005; Shacklock and Brunetto 2011a). Financial circumstances were not included as a predictor of future workplace participation in the present study because (a) finances are well known to enable early retirement or push an employee to continue working, and (b) aged care organizations typically have limited control over this factor (Productivity Commission 2011). Rather, our focus was on the age and health status of employees, and on the employment-related variables of perceived supervisor support and job embeddedness. No prior single study appears to have examined this set of factors in relation to both intention to stay and intention to leave.

\section{Perceived supervisor support}

Perceived Supervisor Support (PSS) refers to employees' perceptions about how much their supervisors care about their wellbeing and value their contribution to the organization (Eisenberger, 2002). Eisenberger (2002) argues that the treatment received by an employee from their supervisor results in that employee feeling a sense of obligation to their supervisor, thereby increasing their intentions to stay with, rather than leave, that organization. While research examining this concept has been conducted primarily in the United States of America (USA) (Rhoades and Eisenberger 2002), some Australian research (O'Donnell and Hudson 2011), as well as research conducted in more highly collectivist nations such as Turkey (Tuzun and Kalemci 2012), has found that supervisor support directly influences turnover. However, limited research has examined the influence of supervisor support on personal care workers' intentions to stay and leave and no research has examined the influence of supervisor support on personal care workers in different aged care 
environments. As the supervision support provided to LTAC and CAC workers differs significantly, supervisor support becomes an important factor to examine when researching retention and turnover intentions of both LTAC and CAC personal care workers.

\section{Job Embeddedness}

Job embeddedness refers to the connections and relationships employees develop over a period of time with their employer and aims to capture a more comprehensive view of the employee-employer relationship than other models of turnover and retention (Holtom and O'Neil 2004; Reitz et al. 2010). USA research examining this construct has found that job embeddedness explained more variance in actual turnover and retention than did either job satisfaction or organizational commitment (Jiang et al. 2012; Reitz et al. 2010). Additionally, job embeddedness has been shown to have a negative relationship with intention to leave across a variety of health workers in the USA (Holtom and O'Neil 2004; Reitz et al. 2010). The strength of connectedness between employees and their organization and communities is argued to be a function of three components: i) the extent to which individuals perceive themselves similar to, or to fit with, their job and community - termed 'fit'; ii) the links individuals have to other people and activities within their organization and community - termed 'links', and iii) the ease with which these links could be broken - the 'sacrifices' employees are willing to make (Mitchell et al. 2001; Reitz et al. 2010). Each component can be further broken into either organization or community effects - that is, on-the-job, or off-the-job, embeddedness.

On-the-job embeddedness research has found that employees who have longer organizational tenures have stronger organizational links and therefore will be less likely to leave (Holtom et al. 2006). Additionally, the more an employee will lose by leaving, the more likely they will stay. Examples of sacrifices employees will make by leaving include the loss of key networks, relationships, friends, projects and organizational benefits (Reitz et al. 2011). Therefore, we expect that community care workers, who spend significant time in the field outside their organization, will report lower levels of on-the-job embeddedness than their long-term care counterparts, who spend the vast majority of time at the one facility. On-the-job embeddedness is thus expected to be a stronger predictor of future workforce participation in LTAC than in CAC workers.

In contrast, off-the-job embeddedness refers to how well employees fit into, and suit, the broader community where they live (Clinton et al. 2012). Factors influencing this perception include weather 
conditions, location, community amenities, political and religious climate, and entertainment activities (Reitz et al 2011; Holtom and Lee 2006). Of relevance to our study, off-the-job embeddedness was found to be more important for nurses than other professions in a USA study of 150 nurses and other workers (Holtom and O'Neil 2004). Additionally, Mitchell et al. (2001) found that, while all three community embeddedness dimensions were negatively related to turnover in hospital workers, only links was related to turnover in a study of grocery workers in the USA. This difference was further supported by Crossley et al. (2007) and Mallol et al. (2007) who found no relationship between offthe-job embeddedness and turnover using a non-health worker sample in the USA. While a metaanalysis found the relationship between off-the-job embeddedness and turnover stronger in collectivist cultures then individualistic cultures (Jiang, Liu, McKay, Lee and Mitchell, 2012), further clarification is still needed of the role that off-the-job embeddedness plays in employee retention and turnover in an Australian context.

To investigate the research questions, the following hypotheses were proposed.

$\mathrm{H} 1$. Older aged care workers have greater intentions to leave and lower intentions to stay in employment than do younger aged care workers.

H2. Aged care workers who are in poor health have greater intentions to leave and lower intentions to stay in employment than do aged care workers in better health.

H3. Intentions to stay increase, and intentions to leave decrease, with (a) perceived supervisor support, (b) on-the-job embeddedness, and (c) off-the-job embeddedness.

H4. Older personal care workers in LTAC settings report higher levels of supervisor support and on-the-job embeddedness, and higher intentions to stay and lower intentions to leave, than those working in CAC settings.

H5. Reasons for intending to stay and leave vary between groups of aged care workers defined by (a) age, (b) health status, and (c) area of employment. For example, perceived supervisor support and on-the-job embeddedness better predict intention to stay and leave among workers in LTAC than in those working in CAC.

\section{Methods}


Ethics approval was received from the university and each of four Australian aged care organizations (1 very large (>4000 employees), 1 large (<900 employees), 1 medium (<300 employees), 1 small (<80 employees)) spread across rural, regional and metropolitan settings over two states of Australia. Invitations to participate were sent to 2118 employees of these organizations. To be eligible for inclusion in this study, employees needed to be working as a direct care worker (providing front-line care services to clients). Follow-up emails were sent at two and four weeks after questionnaires were sent. Surveys were distributed in bulk to each site by the researcher, based on total numbers of direct care employees provided by each organisation. Survey packs were placed in staff/common rooms as allocated by site management, to be completed and returned to the researcher using the envelope provided or placed in a sealed box at site. Once the questionnaires were returned, the responses were entered into SPSS Version 21.0 for analysis.

\section{Measures}

The questionnaire contained items regarding the respondent's gender, age, health status and area of employment. To measure participants' health, we asked, "How would you rate your health?" on a 6point scale (very poor, poor, fair, good, very good, excellent). Respondents were asked to identify the main area of aged care work: "What area of aged care do you primarily work in?" The response options were Home Care (CAC), or Residential Care (LTAC). Employees responded to the remaining items, except those listed below under organizational and community links, on a 5-point Likert scale ( $1=$ strongly disagree, to $5=$ strongly agree) .

Perceived Supervisor Support (PSS) was measured using a version of the Shortened Perceived Organizational Support Scale, that was adapted by replacing the words "your organization" with "your supervisor", in line with previous research examining PSS, such as Kottke and Sharafinski (1988) and Eisenberger et al. (2002). This scale consists of 16 statements that represent opinions employees may have about their organization, rated on a 7-point scale ranging from strongly disagree (1) to strongly agree (7). The measure has been found to be a reliable $(\alpha=.93)$ and valid measure of PSS in previous studies (DeConinck and Johnson 2009; Shanock and Eisenberger 2006; Eisenberger et al. 2002). A composite score was used in the final analysis by averaging responses to items in this scale. An example of the items used was, 'My immediate supervisor strongly considers my goals and values'. 
Job Embeddedness was measured using Lee et al.'s (2004) measure of Job Embeddedness, consisting of 34 items relating to the fit, links and sacrifices employees perceive with their organization and broader community. Employees responded to the 'links' items as either yes, no or not applicable. This study examined both On-the-job and Off-the-job embeddedness by creating a composite score for both scales, as per previous research by Lee et al. (2004). An example of the organization (on-the-job) embeddedness items used is, 'The benefits are good on this job'. An example of the community (off-the-job) embeddedness items used is, "I really love the place where I live'.

Intention to Stay (dependent variable) is the perception of the "estimated likelihood of continued membership in an organization" (Price and Mueller 1981 p. 546). It was measured using four items developed by Kim et al. (1996). An example of the items used is: 'I plan to stay at this organization for as long as possible'. Intention to Leave (dependent variable) was measured using four items adapted from Bradley (2007). These items included: 'Over the past month, I have seriously thought about resigning from the workforce altogether'. Bradley reports the scale to have adequate reliability (Cronbach's alpha $=.79$ ), and concurrent validity in the form of moderate to high correlations with job demands and job stress.

The first step in the data analyses was a principal axis factor analysis to verify that the set of eight items designed to measure intention to stay and intention to leave actually load separately on these two constructs. Following oblique rotation and removal of one cross-loading intention to stay item, a clean solution was obtained with two factors each having eigenvalues greater than unity and $57 \%$ of the variance explained. All seven items loaded in excess of .62 on their intended factor, and no item cross-loaded at greater than .12 . The responses to the four intention to leave items, and the responses to the three remaining intention to stay items, were separately averaged to form composite scales measuring these two dependent variables.

\section{Results}

A total response rate of $17 \%$ was achieved (359 useable responses). This paper reports the responses of the 206 personal care workers from within this sample. Reasons for this modest 
response rate are unknown, but the response rate could have been affected by the national census of Australian aged care employees, which was being conducted at the same time as this study. Other possible reasons include concerns about the confidentiality of responses from employees, or practical problems with the distribution and return of the questionnaire. Of these respondents, $195(94.7 \%)$ were females and 11 (5.3\%) were males. Furthermore, 150 were aged over 45 years $(73.5 \%), 54$ were aged under 45 years (26.5\%) ( $M=55$ years: SD: 6 years), 69 (46\%) worked in long-term care, and 81 (54\%) worked in community care.

Table 1 provides descriptive statistics and correlations for all variables. As shown, the respondents reported moderately low turnover intentions, as indicated by a mean level of intention to stay that was above the scale mid-point and a mean intention to leave that was below the scale midpoint. The two dependent variables were moderately, negatively correlated $(r=-.48)$. The three main predictors (perceived supervisor support, on-the-job embeddedness, and off-the-job embeddedness) were significantly correlated, in the expected directions, with both intention to stay and intention to leave. However, contrary to expectations, age and health were unrelated to the two intention variables. Therefore Hypothesis 3 was supported, but Hypotheses 1 and 2 were not. Following a split of the sample by age ( $<45$ years vs. 45 years and older), independent samples t-tests confirmed that there was no significant difference by age group in intention to leave $(t=-.65, d f=204, p=.52)$, or intention to stay $(t=-.04, d f=204, p=.97)$.

\section{(Table 1 about here)}

Intentions to stay and leave varied by area of employment, although not in the directions predicted in Hypothesis 4. Therefore, the remaining analyses compared the responses of CAC workers and LTAC workers. To focus on older aged care workers, analyses were restricted to respondents aged 45 years and over $(N=150)$, representing older workers as per Australian Bureau of Statistics (2011) criteria. Descriptive statistics, and correlations between the key study variables, by area of employment, are given in Table 2. Independent samples t-tests revealed that the two groups differed for perceived supervisor support, intention to stay and intention to leave, but not for either job embeddedness factor. These findings do not support Hypothesis 4: differences between the two worker groups either did not exist or were in the opposite direction to that predicted. Comparison of the bi-variate correlations between the two sub-samples revealed no significant differences, although 
the correlation between supervisor support and intention to stay was considerably higher in the group of LTAC workers $(r=.41)$ than in the CAC workers $(r=.22, p>.05)$, consistent with Hypothesis 5.

\section{(Table 2 about here)}

Two regression analyses were performed, with intention to leave and intention to stay, respectively, as the criteria. As recommended by Cohen et al. (2003) the predictors were added to the regression equations in a hierarchical manner so as to enable the effects of the interactions between employment area and the other organizational variables to be estimated separately from their main effects. Specifically, predictors were entered in three steps: 1. area of employment (LTAC or CAC), 2. perceived supervisor support, on-the-job embeddedness and off-the-job embeddedness, and 3. the three interaction terms, represented by the product of area of employment and each of the three core predictors. A significant change in $R^{2}$ at step 3 would indicate that one or more of the predictors differentially influenced intention to stay or leave in the two groups of workers. However, in neither case did the third step result in a significant increment in $R^{2}$, and none of the individual interaction terms approached significance (all ps $>.20$ ). Therefore, in Table 3, results from these analyses are reported after the second step, with just the main effects included in the equations.

\section{(Table 3 here)}

As can be seen, the model predicting older workers' intentions to leave explained $31 \%$ of the variance in this criterion, $p<.001$. At step 1 , area of employment explained $5.5 \%$ of the variance, $p<.001$. At step 2, entry of the three core predictors accounted for an additional $25.4 \%$ of the variance $(p<.001)$. After this step, perceived supervisor support and on-the-job embeddedness were significant, whereas area of employment and off-the-job embeddedness were not. In contrast, the model predicting intentions to stay explained $17 \%$ of the variance, $p<.001$. At step 1 , area of employment was significant, explaining $4.0 \%$ of the variance, $p<.001$. At step 2 , entry of the core predictors accounted for an additional $12.2 \%$ of the variance in the criterion $(p<.001)$, with both perceived supervisor support and on-the-job embeddedness significant, and area of employment and off-the-job embeddedness not so. Thus, the pattern of significant predictors was the same for both criteria, although substantially more of the variance in intention to leave than in intention to stay was explained by this predictor set. 


\section{Discussion}

This study revealed several important and interesting findings for aged care management. First, unexpectedly, employees' age and health were not significant predictors of retention and turnover intentions. While this finding contrasts with previous literature by Reitz et al. (2010) and Shacklock et al. (2011b), those studies examined nurses within an acute-care sector, whereas our study examined personal care workers in an aged care sector, indicating that context and position differences may account for these variances. One explanation could be that personal care workers find sufficient meaning and value in their jobs to override the common turnover influences of age and health. Another explanation could be that health challenges presented as employees' age are not similarly perceived by personal care workers. Perhaps physical demands are better managed by care workers compared to other jobs, or are perceived to be more amenable to older workers remaining working. Alternatively, aged care workers may have fewer alternative employment opportunities than more highly skilled workers to obtain less burdensome roles. Whatever the reason, as this research was the first to examine these influences upon personal care workers; further research is required to identify if the findings are a result of role and context variances, or other factors.

Our research also revealed that CAC personal care workers reported higher intentions to stay, lower intentions to leave and higher perceived supervisor support than those within LTAC settings. This finding suggests greater stability in employment and satisfaction with supervision arrangements within CAC settings, supporting King et al. (2012), who found CAC workers were more satisfied than LTAC workers with their current job conditions. Future research could usefully investigate the extent to which differences between LTAC and CAC workers are due to the kinds of people employed in these sectors, as opposed to differences in the kinds of jobs they perform and/or the work environments. While none of the current set of variables differentially predicted staying or leaving in the LTAC and CAC groups, this may have partly been due to a lack of statistical power. Should the study be replicated with a larger sample, current findings suggest that perceived supervisor support, in particular, may be differentially predictive of intentions to stay in the two groups. Combined, these points suggest that, while supportive supervision may encourage retention in LTAC environment $s$ (where supervision is typically close), this may be less relevant in CAC contexts. Further research is required to test these propositions. 
This study found that area of employment, supervisor support and on-the-job embeddedness were all significant predictors of both older workers' intentions to stay and leave, although the strength of the predictive relationships differed. In particular, the variables explained more variance in intentions to leave than to stay. This suggests that while there is some overlap in the motivators of employee retention and turnover, there are also some differences. Future studies should therefore seek to uncover variables more important to retention than to turnover. Variables both within (e.g., co-worker support) and external to the organization (e.g., labour market opportunities) may help improve the prediction of intentions to stay. Interestingly, our research found that off-the-job (community) embeddedness did not influence older employees' intentions to stay or leave, contrasting with previous studies (Holtom and Lee 2006; Reitz et al. 2011) in USA healthcare. A possible contributing factor is that, for many workers, leaving their job does not require re-locating their place of residence to a different community. However, further comparative research is required to examine this.

\section{Implications for Nursing Management}

Unexpectedly and importantly, personal care workers' age and health were not related to intentions to stay or leave employment. The implications for management include the positive targeting of older workers for sustained employment within aged care, with associated expectations for higher retention than for older workers in other sectors. Further, due to increasing reliance on older workers - the growing labour market share - healthcare organizations/managers must encourage new approaches for retaining employees via supervisor support. The literature contains examples of interventions designed to enhance workplace supervisor support (e.g., Heaney et al. 1995) plus reviews of the field (e.g., Mitchell et al. 1982). Employing older workers need not mean short-lived working futures, as it may in other sectors/industries where younger workers typically leave organizations earlier than older workers. Thus, to retain their workforce, our findings suggest that aged care managers foster relationships with personal care workers, and encourage workers to establish and maintain strong links within the organization, plus ensure workers fit the organization/unit. As shown, different retention strategies may be required for LTAC and CAC workers.

Additionally, there is now an evidence-based need for managers to embrace flexible human resource practices to retain an age-diverse workforce. Several factors were found to affect all personal care workers' intentions to leave and stay, and these should be the centrepiece of retention strategy 
development. This is particularly salient in an industry which is growing exponentially to meet the demands of an ageing population (Productivity Commission 2011), and where high turnover has been linked to poorer health outcomes.

\section{Strengths and Limitations}

Even though our results make important contributions, the study was not without limitations. We used a cross-sectional survey design to collect data and therefore we acknowledge that while relationships have been identified, these do not indicate causation. Additionally, while this study had an adequate sample size, generalisation of findings outside the sample must be made with caution. Further, Australian aged care might be different from other contexts because it is dominated by females and offers flexible working arrangements (known to be attractive to older workers), and therefore further research is warranted in other contexts.

\section{Conclusion}

Our study investigated predictors of turnover and retention intentions of personal care workers within the Australian aged care sector. We found that perceived supervisor support, on-the-job embeddedness and area of employment all significantly influenced these workers' intentions to stay and leave employment. Although the set of predictors explained more variance in intention to leave than intention to stay, the study identified similarities between retention and turnover motivators, as well as some potential differences that need to be acknowledged. Additionally, this study found that personal care workers working within a CAC environment were more likely to stay and reported higher supervisor support than those working within a LTAC environment.

Creating and maintaining an efficient, qualified workforce is a central requirement of any organization; however, within an ageing workforce context, retaining older workers is of particular importance. Therefore, research clarifying the factors that influence older workers' intentions to stay and leave is crucial to organizations throughout the world. Further research would be worthwhile examining other aged care employees, in other countries, to test the generalizability of the current findings. 


\section{References}

Australian Bureau of Statistics (ABS). (2011) Retirement and retirement intentions, Australia, July 2010 to June 2011. Catalogue 6238.0 Australian Bureau of Statistics, Canberra.

Australian Institute of Health and Welfare (AlHW). (2010) Australia's health series 12. Cat no. Aus 122 Australian Government: Canberra.

Australian Institute of Health and Welfare (AlHW). (2012) Long term aged care in Australia 20102011: A statistical overview. Aged care statistics series no. 36. Cat. no. AGE 68. Canberra: AlHW.

Bradley G. L. (2007) Job tenure as a moderator of stressor-strain relations: A comparison of experienced and new-start teachers. Work and Stress 21 (1), 48-64.

Chan E-Y. and Morrison P. (2008) Factors influencing the retention and turnover intentions of registered nurses in a Singapore hospital. Nursing and Health Sciences 2 (2) 113-121.

Cheung J. (2004) The decision process of leaving nursing. Australian Health Review 28 (3), 340-348.

Clinton M, Knight T. and Guest D. (2012) Job embeddedness: A new attitudinal measure. International Journal of Selection and Assessment 20 (1), 111-117.

Cohen J, Cohen P, West S. G. and Aiken, L. (2003) Applied multiple regression/ correlation analysis for the behavioral sciences ( ${ }^{\text {rd }}$ ed.), Erlbaum, Hillsdale, New Jersey.

Coomber B. and Barriball L. K. (2007) Impact of job satisfaction components on intent to leave and turnover for hospital-based nurses: A review of the research literature. International Journal of Nursing Studies 44 (2), 297 - 314.

Crossley C. D., Bennett R. J., Jex S. M. and Burnfield J. L. (2007) Development of a global measure of job embeddedness and integration into a traditional model of voluntary turnover. Journal of Applied Psychology 92 (4), 1031-1042.

DeConinck J. and Johnson J. (2009) The effects of perceived supervisor support, perceived organizational support, and organizational justice on turnover among salespeople. Journal of Personal Selling and Sales Management 29 (4) 333 - 351.

Department of the Treasury. (2004) Australia's Demographic Challenges. Canberra: Department of the Treasury.

Department of Health and Ageing. (2004) Best practice approaches to minimise functional decline in the older person across the acute, subacute an long term aged care settings. Clinical Epidemiology and Health Service Evaluation Unit. Melbourne. Retrieved 18/5/2010 at http://www.health.vic.gov.au/acute-agedcare/functional-decline-manual.pdf 
Department of Health and Ageing. (2005) National aged care workforce strategy. Canberra:

Department of Health and Ageing.

Department of Health and Ageing. (2011a) Help to stay at home - community care. Retrieved 6 June 2011 at: http://www.health.gov.au/internet/main/publishing.nsf/Content/ageing-commcareindex.htm-copy3

Eisenberger R., Stinglhamber F., Vandenberghe C., Sucharski I. L. and Rhoades L. (2002) Perceived supervisor support: Contributions to perceived organizational support and employee retention. Journal of Applied Psychology 87 (3), 565-573.

Eley R., Eley D. and Rogers-Clarke C. (2010) Reasons for entering and leaving nursing: an Australian regional study. Journal of Advanced Nursing 28 (1). 6-13.

Encel S. and Studencki H. (2004) Older workers: Can they succeed in the job market? Australasian Journal on Ageing 23 (1), 33-37.

Ferres N., Travaglione A. and Firns I. (2003) Attitudinal differences between Generation X and older employees. International Journal of Organizational Behavior 6 (3), 320-333.

Gelesma T., Ven Der Doef M., Maes S., Janssen M., Akerboom S. and Verhoeven C. (2006) A longitudinal study of job stress in the nursing profession: Causes and consequences. Journal of Nursing Management 14 (4), 289-299.

Heaney C. A., Price R. H. and Rafferty J. (1995) Increasing coping resources at work: A field experiment to increase social support, improve team functioning, and enhance employee mental health. Journal of Organizational Behavior 16 (4), 335- 352.

Holtom B. C., Mitchell T. R. and Lee T. W. (2006) Increasing human and social capital by applying job embeddedness theory. Organizational Dynamics 35 (4), 316 - 331.

Holtom B. C. and O'Neill B. S. (2004) Job Embeddedness: A theoretical foundation for developing a comprehensive nurse retention plan. Journal of Nursing Administration 34 (5), 216-227.

Jackson N., Walter, M., Felmingham, B. and Spinaze, A. (2006) Will older workers change their retirement plans in line with government thinking? A review of recent literature on retirement intentions. Australian Labour Bulletin 32 (4), 315-344.

Jiang K., Liu D., McKay P., Lee T. and Mitchell T. (2012) When and how is job embeddedness predictive of turnover? A meta-analytic investigation. Journal of Applied Psychology 97 (5), 1077-1096

Kim S-W., Price J L., Mueller C W. and Watson T. W. (1996) The determinants of career intent among physicians at a U.S. Air Force hospital. Human Relations 49 (7), 947-976

King D., Mavromaras K., Wei Z., He B., Healy J., Macaitis K., Moskos M. and Smith L. (2012) The Aged Care Workforce, 2012. Canberra: Australian Government Department of Health and Ageing. 
Kottkey J. L. and Sharafinski C. E. (1988) Measuring perceived supervisory and organizational support. Educational and Psychological Measurement 48 (4), 1075-1079.

Lambert E. and Hogan N. (2009) The importance of job satisfaction and organizational commitment in shaping turnover intent: A test of a causal model. Criminal Justice Review 34 (1), 96-18.

Lee T. W., Mitchell T. R., Sablynski C. J., Burton J. P. and Holtom B. C. (2004) The effects of job embeddedness on organizational citizenship, job performance, volitional absences and voluntary turnover. Academy of Management Journal 47 (5), 711-722.

McCarthy G., Tyrrell M. P. and Lehane E. (2007) Intention to 'leave' or 'stay' in nursing. Journal of Nursing Management 15 (3), 248-255.

Mallol C., Holtom B. and Lee T. (2007) Job Embeddedness in a Culturally Diverse Environment. Journal of Business and Psychology 22 (1), 35-44.

Mitchell R. E., Billings A. G. and Moos R. H. (1982) Social support and well-being: Implications for prevention programs. Journal of Primary Prevention 3 (2), 77-98.

Mitchell T. R., Holtom B. C., Lee T. W., Sablynski C. J. and Erez M. (2001) Why people stay: Using job embeddedness to predict voluntary turnover. Academy of Management Journal 44 (6), 1102-1121.

Mittal V., Rosen J. and Leana C. (2009) A dual-driver model of retention and turnover in the direct care workforce. The Gerontologist 49 (5), 623-634.

Morrell K., Loan-Clarke J., Arnold J. and Wilkinson A. (2008) Mapping the decision to quit: A refinement and test of the unfolding model of voluntary turnover. Applied Psychology: An International Review 57 (1), 128-150.

O'Donnell J. and Hudson C. (2011) Practitioner introduction healthcare reform: Government pronouncements or managers engaging staff? Asia Pacific Journal of Human Resources 49 (2), 138 - 142.

OECD. (2006). Live longer. Paris: Organization for Economic Co-operation and Development.

O'Loughlin K., Humpel N. and Kendig H. (2010) Impact of the global financial crisis on employed Australian baby boomers: A national survey. Australasian Journal on Ageing 29 (2), 88-91.

Palese A., Pantali G. and Saiani L. (2006) The management of a multigenerational nursing team with differing qualifications: A qualitative study. The Health Care Manager 25 (2), 173-193.

Phillipson C. (2004) Work and retirement transitions: Changing sociological and social policy contexts. Social Policy and Society 3 (2), 155-162.

Price J. L. and Mueller C. W. (1981) A causal model for turnover for nurses. Academy of Management Journal 24 (3), 543-565. 
Productivity Commission. (2005) Economic implications of an ageing Australia Research report. Canberra: Australian Government Productivity Commission.

Productivity Commission. (2011) Care for older Australians (pp. 1-574). Canberra: Productivity Commission.

Radford K., Shacklock KH. and Bradley G. (2012) Battle of the decades: Generational differences in the retention of Australian aged care employees. In Emerging Researcher In Ageing Conference Proceedings. Brisbane, 19-20th November 2012.

Reitz O., Anderson M. and Hill P. (2010) Job embeddedness and nurse retention. Nurse Administration Quarterly 34 (3), 190-200.

Rhoades L. and Eisenberger R. (2002) Perceived Organizational Support: A Review of the Literature. Journal of Applied Psychology 87 (4), 698-714.

Schmieder R. A. and Smith C. S. (1996) Moderating effects of social support in shiftworking and nonshiftworking nurses. Work and Stress 10 (2), 128-140.

Shacklock K. and Brunetto Y. (2011a) A model of older workers' intentions to continue working. Personnel Review 40 (2), 252-274.

Shacklock K. and Brunetto Y. (2011b) The intention to continue nursing: Work variables affecting three nurse generations in Australia. Journal of Advanced Nursing 68 (1), 36-46.

Shanock L. R. and Eisenberger R. (2006) When supervisors feel supported: Relationships with subordinates' perceived supervisor support, perceived organizational support, and performance. Journal of Applied Psychology 91 (3), 689-695.

Tourangeau A.E., Cummings G., Cranley L.A., Ferron E.M. and Harvey S. (2009) Determinants of hospital nurse intention to remain employed: broadening our understanding. Journal of Advanced Nursing 66 (1), 22-32.

Tuzun I. K. and Kalemci R. A. (2012) Organizational and supervisory support in relation to employee intentions. Journal of Managerial Psychology 27 (5), 518-534. 